\title{
The role of CEUS in characterization of superficial lymph nodes: a single center prospective study
}

\section{Giorgio de Stefano${ }^{1}$, Umberto Scognamiglio', Filomena Di Martino², Roberto} Parrella $^{3}$, Francesco Scarano ${ }^{3}$, Giuseppe Signoriello ${ }^{4}$ and Nunzia Farella

${ }^{1}$ UOC Malattie Infettive ad Indrizzo Ecointerventistico -AORN dei Colli, Naples, Italy

2 UOC Infezioni Sistemiche e dell' Immunodepresso -AORN dei Colli, Naples, Italy

${ }^{3}$ UOC Malattie Infettive ad Indirizzo Respiratorio -AORN dei Colli, Naples, Italy

${ }^{4}$ SUN Napoli- Dipartimento Salute Mentale, Servizio Statistica Medica, Naples, Italy

Correspondence to: Giorgio de Stefano, email: giordest1@virgilio.it

Keywords: CEUS, lymph nodes, contras enhanced ultrasound, metastases

Received: March 16, 2016

Accepted: May 05, 2016

\section{ABSTRACT}

Accurate lymph node characterization is important in a large number of clinical settings. We evaluated the usefulness of-Contrast Enhanced Ultrasound (CEUS) in distinguishing between benign and malignant lymph nodes compared with conventional ultrasonography in the differential diagnosis of superficial lymphadenopathy.

We present our experience for 111 patients enrolled in a single center.

111 superficial lymph nodes were selected and only 1 lymph node per patient underwent CEUS. A definitive diagnosis for all lymph nodes was obtained by ultrasonographically guided biopsy and/Orexcision biopsy.

The size of the lymph nodes, the site (neck, axhla, inguinal region) being easily accessible for biopsy, and the US and color Doppler US characteristics guided us in selecting the nodes to be evaluated by GEUS.

In our study we identified different enhancement patterns in benign and malignant lymph nodes, with a-high degree of diagnostic accuracy for superficial lymphadenopathy in comparison with conventional US.

\section{INTRODUCTIQ}

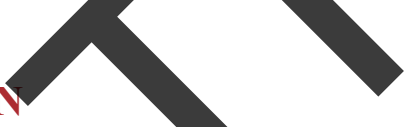

Accurate lymph node characterization, including the ability to distinguish benign from malignant lymphadenopathy [1], is important in a large number of clinical settings,

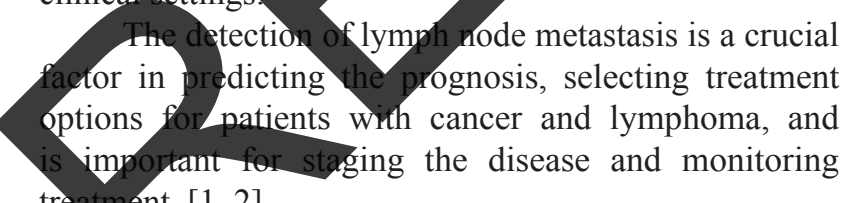
treatment. $[1,2]$

Since a clinical diagnosis of superficial lymphadenopathy is limited, different diagnostic imaging techniques are used to characterize lymph nodes. Ultrasonography (US) is the most commonly used method for evaluating superficial lymph nodes. It is widely available, easy to use and less expensive than other imaging methods, and it has the benefit of not involving ionizing radiation. $[3,4]$
Unlike other imaging modalities, such as computed tomography (CT) and magnetic resonance imaging (MRI), which mainly depend on the size of the node for a differential diagnosis, gray-scale ultrasonography combined with Doppler examination can be used to assess important parameters such as the shape, border, internal structure, calcification, necrosis, echogenicity and vascularization of lymph nodes. [3, 4, 5]

Recent advances in ultrasound technology, including ultrasonographic contrast agents, contrastspecific ultrasonographic modes, and the development of specific software for quantification, have shown potential to improve the accuracy of ultrasonography and for the diagnosis of superficial lymphadenopathy. $[6,7]$

Contrast-enhanced ultrasound (CEUS) is a modern imaging method that evaluates tissue perfusion in real time. It offers better prospects for an objective evaluation.

Several authors in the literature show data on the use of CEUS for the characterization of enlarged 
Table 1: Lymph node characterization by Doppler

\begin{tabular}{|l|l|l|l|}
\hline & $\begin{array}{l}\text { Benign } \\
\text { Lymph- } \\
\text { nodes } \\
\text { (N.61) }\end{array}$ & $\begin{array}{l}\text { Malignant } \\
\text { Lymph- } \\
\text { nodes } \\
\text { (N.50) }\end{array}$ & $\begin{array}{l}\text { Total } \\
\text { (N.111) }\end{array}$ \\
\hline $\begin{array}{l}\text { Category 1 : } \\
\text { Hilar pattern with flow signals in the nodal hilum }\end{array}$ & $44(73.1 \%)$ & $15(30.0 \%)$ & $59(53.1 \%)$ \\
\hline $\begin{array}{l}\text { Category 2 } \\
\text { Peripheral pattern with flow signals mainly in } \\
\text { peripheral nodal parts }\end{array}$ & $4(6.6 \%)$ & $12(24.0 \%)$ & $16(14.4 \%)$ \\
\hline $\begin{array}{l}\text { Category 3 } \\
\text { Mixed pattern with both a hilar and peripheral } \\
\text { pattern }\end{array}$ & $5(8.2 \%)$ & $16(32.0 \%)$ & $21(18.9 \%)$ \\
\hline $\begin{array}{l}\text { Category 4 } \\
\text { No flow pattern with absence of signal }\end{array}$ & $8(13.1 \%)$ & $7(14.0 \%)$ & $15(13.5 \%)$ \\
\hline
\end{tabular}

superficial lymph nodes. In this study, we evaluated the usefulness of CEUS in distinguishing between benign and malignant lymph nodes compared with conventional ultrasonography in the differential diagnosis of superficial lymphadenopathy. We present our experience with 111 patients enrolled in a single center.

\section{MATERIALS AND METHODS}

Between September 2013 and November 2015, 111 patients with enlarged superficial lymph nodes y examined at our Interventional Ultrasound Unit- AOR dei Colli-Naples-Italy. The majority of these subject were referred for US examination due to the existence of palpable superficial masses. Forty-eight p were male and $63(56.3 \%)$ were female, and the mean age was 44 years (range 18-71 years). Twenty patients were African and 90 Caucasian. Fiftymine cases $(53.6 \%)$ had nodes in the neck, $29(26.3 \%)$ had nodes in the axilla and 22 subjects $(20 \%)$ had noos in the inguinal region. Among these patients, 2 had laryngitic cancer, 4 had breast cancer and 3, had lymphoma. The other 92 cases had lymphadenopathy of an uncertain nature without any history of neoplastic disease. Thirty-eight subjects were HIV-positiye

We selected 111 superficial lymph nodes, and only 1 lymph node perpatient underyen CEUS. The size of the lymph nodes, the site (neck, axilla, inguinal region) being sily access ble for biopsy, and the US and color Doppler US characteristics guided us in selecting the nodes to be evaluated by CEUS.

CEUS was performed by three experienced

Operators with more than 15 years' experience.

This study was approved by the Local Ethics Committee, and informed consent was obtained from all patients enrolled.

A commercially available ultrasound scanner (Aloka ProSound Alpha 10) was used with a high-frequency linear array probe $(6-15 \mathrm{MHz})$. The longitudinal to transverse $(\mathrm{L} / \mathrm{T})$ (Rounded index or Solbiati Index) nodal ratio was determined using conventional ultrasonggraphy: it was defined as the atio between the longest diameter of the node and the length of the node in a perpendicular plane. The marsins (sharp, irregular, blured), shape, internal echogenicity (hypoechoic, isoechoic, hyperechoic) and hilum visibility (presence or absence of a central ogenic hilum of the lymph node) were noted.

Indications of malignancy as detected with conventional ultrasonography included a minimum transverse dianteter greater than $7 \mathrm{~mm}$, an $\mathrm{L} / \mathrm{T}$ ratio of 2 or less, heterogeneous echogenicity, irregular borders, and echo incompleteness in the hilum. A peripheral or mixed flow was considered a sign of malignancy. When the minimum transverse diameter of the lymph node was greater than $10 \mathrm{~mm}$, the shape was round or teardrop-like, the $\mathrm{L} / \mathrm{T}$ was 2 or less, the echogenic hilum was not present or widened eccentrically, and the color Doppler showed flow only in the hilum, it was considered malignant.

In Doppler US (pulse repetition frequency $350 \mathrm{~Hz}$, wall filter $45 \mathrm{~Hz}$ ) four patterns of nodal vascularization were defined: 1) a hilar pattern with flow signals in the nodal hilum, 2) a peripheral pattern with flow signals mainly in peripheral nodal parts, 3) a mixed pattern with both a hilar and peripheral pattern and 4) no flow pattern with absence of signal.

Color Doppler US showed different patterns in malignant versus benign nodes ( Table 1).

$73.7 \%$ of the benign nodes had hilar and regular vascular pattern, with only one pedicullum (44 of 32 nodes). More than half of the malignant nodes had peripheral or mixed vessels with chaotic patterns and multiple pedicullus.

\section{CEUS EXAMINATION}

Pulse-inversion harmonic CEUS using the contrast/general preset and a low mechanical index (0.06) was performed and recorded for approximately 120 seconds. The ultrasonographic contrast agent SonoVue (BraccoSpA, Milan, Italy) was used in the examinations. 
Table 2: Lymph node characterization by CEUS

\begin{tabular}{|l|l|l|l|}
\hline & $\begin{array}{l}\text { Benign } \\
\text { Lymph- } \\
\text { nodes } \\
\text { (N.61) }\end{array}$ & $\begin{array}{l}\text { Malignant } \\
\text { Lymph- } \\
\text { nodes } \\
\text { (N.50) }\end{array}$ & $\begin{array}{l}\text { Total } \\
(\mathbf{N . 1 1 1})\end{array}$ \\
\hline $\begin{array}{l}\text { Category 1 : } \\
\text { Intense homogeneous enhancement }\end{array}$ & $45(73.7 \%)$ & $7(14.0 \%)$ & $52(46.8 \%)$ \\
\hline $\begin{array}{l}\text { Category 2 } \\
\text { Moderate homogeneous enhancement }\end{array}$ & $7(11.5 \%)$ & $7(14.0 \%)$ & $14(12.6 \%)$ \\
\hline $\begin{array}{l}\text { Category 3 } \\
\text { Inhomogeneous enhancement }\end{array}$ & $8(13.1 \%)$ & $32(64.0 \%)$ & $40(36.0 \%)$ \\
\hline $\begin{array}{l}\text { Category 4 } \\
\text { No enhancement }\end{array}$ & $1(1.6 \%)$ & $4(4.5 \%)$ & $5(4.5 \%)$ \\
\hline
\end{tabular}

A bolus of $2.4 \mathrm{~mL}$ of SonoVue contrast agent was administrated followed by injection of $5 \mathrm{~mL}$ of a physiologic saline solution and video clips were recorded in the first 3 minutes. All patients gave their informed consent for the intravenous administration of the contrast agent. A $2.4 \mathrm{~mL}$ dose of the contrast agent was bolus injected into a peripheral vein, followed by injection of 5 $\mathrm{mL}$ of a physiologic saline solution. The nodal perfusion and the enhancement pattern were evaluated in the arterial phase (10-15s after bolus of contrast agent) and parenchymal phase (15-30s after bolus of contrast agent).

The pattern of lymph node perfusion, as determined using CEUS, was divided into 4 qualitative categories category 1 included lymph nodes that showed inte homogeneous enhancement; category 2 included lymp nodes with a moderate homogeneous enhancement category 3 included lymph nodes that showed inhomogeneous enhancement; category 4 included lymph nodes that lacked any detectable contrast enhancement.

A definitive diagnosis

obtained by ultrasonographically guided biopsy and/or excision biopsy.

The results obtained by CEUS examination are summarized in Table

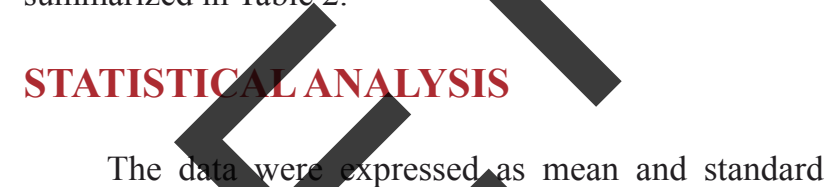
deviation. The statistical analysis was performed using SPSS software version 21.0 (JBM SPSS 21).

ROQ (Receiver Operating Characteristic) analysis vas used to assess the diagnostic value of four patterns of lynph node perfusion. To identify the cut-off levels of ultrasound perfusion the area under the ROC curve was used. Subsequently, the threshold value identified indicated the sensitivity, specificity and predictive values of the test and $95 \%$ confidence intervals of the estimate.

ROC curves were compared with DeLong's test for two correlated ROC curves to compare the AUC of the CEUS and Doppler test.

\section{RESULTS}

The histological results showed that 61 of the 111 enlarged lymph nodes were benign (i.e., 45 reactive and 16 tubercular lymph nodes) and 50 ymph nodes were malignant (31 metastases, 19 lymphomas). The CEUS findings indicated 52 lymph nodes in category 1 (37 benign, 81ymphomas and 5 tubercular lymph nodes), 14 category 2 ( 6 benign, 2 metastatic, 5 lymphomas and tubercular), 40 in category 3 (1 benign, 27 metastatic, 4 lymphomas and 8 tubercular lymph nodes ), and 5 in category 4 (1 benign, 2 lymphomas, 1 malignant and 1TB.

Reactive and tuberculosis lymph nodes were ered benign while metastatic and lymphoma lymph nodes were considered malignant.( Table 2 )

When lymph nodes in categories 1 and 2 were diagnosed as benign and lymph nodes in categories 3 and 4 were diagnosed as malignant, the sensitivity, specificity, and accuracy levels for CEUS were $72 \%, 85.2 \%$ and $80 \%$, and $46.0 \%, 78.6 \%$ and $63.0 \%$ for Doppler. (Table 3 )

The comparison between the AUC of the two tests was statistically significant $(p<0.001)$ (Figure 5). The heterogeneity of behavior at CEUS of tubercular and lymphoma lymph nodes greatly reduces overall the sensitivity, specificity and diagnostic accuracy in the characterization of lymphadenopathy.

\section{DISCUSSION}

Conventional ultrasonography is recognized as a valuable means to evaluate superficial lymph nodes.[7, 8, 9]

The use of high-frequency transducers allows an accurate evaluation of the morphologic characteristics and structure of lymph nodes, and contrast agents allow a better description of the microvascular pattern and can detect avascular areas of necrosis and tumor metastasis. $[9,10]$

Rubaltelli et al reported the limitation of US and color Doppler sonography in depicting partial metastases, i.e. cases in which only part of the node has been replaced 

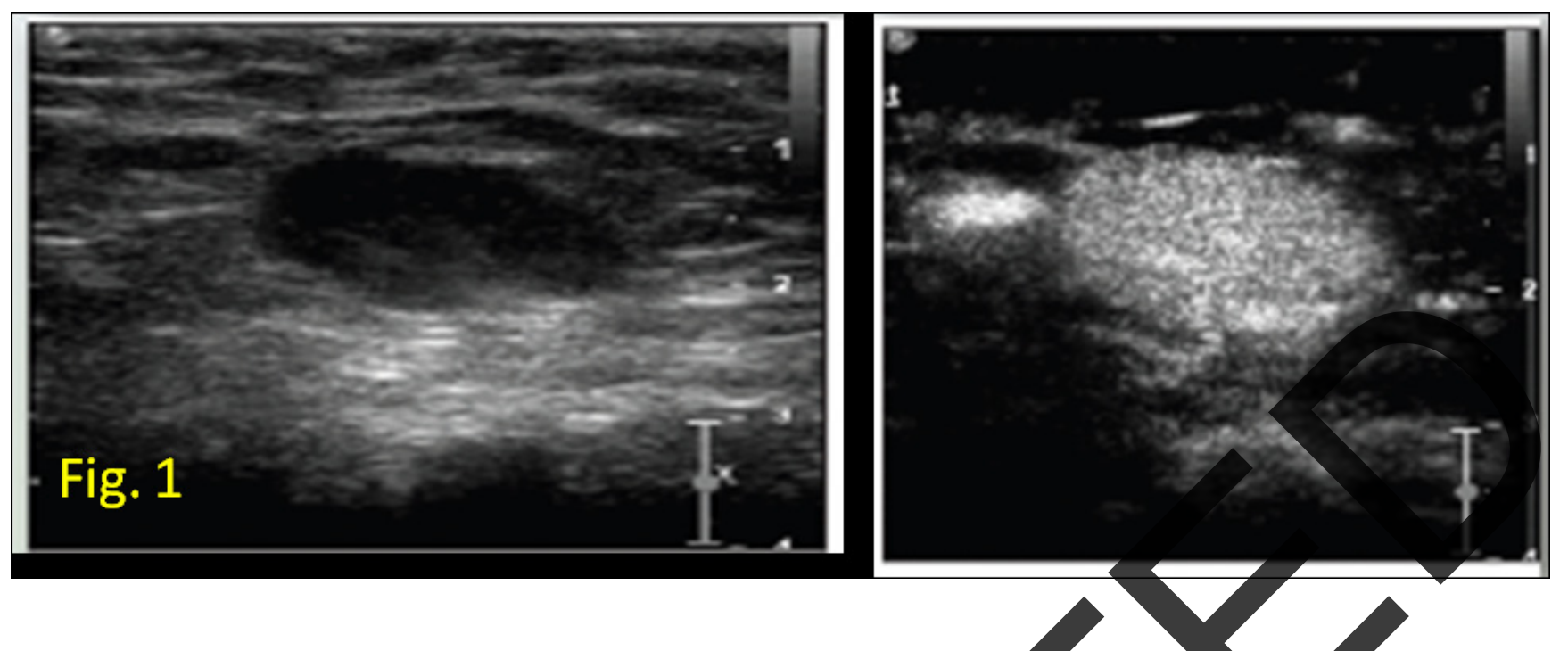

Figure 1: Reactive lymph node: CEUS shows an intense, centripetal, rapid and uniform enhancement in the arterial phase.

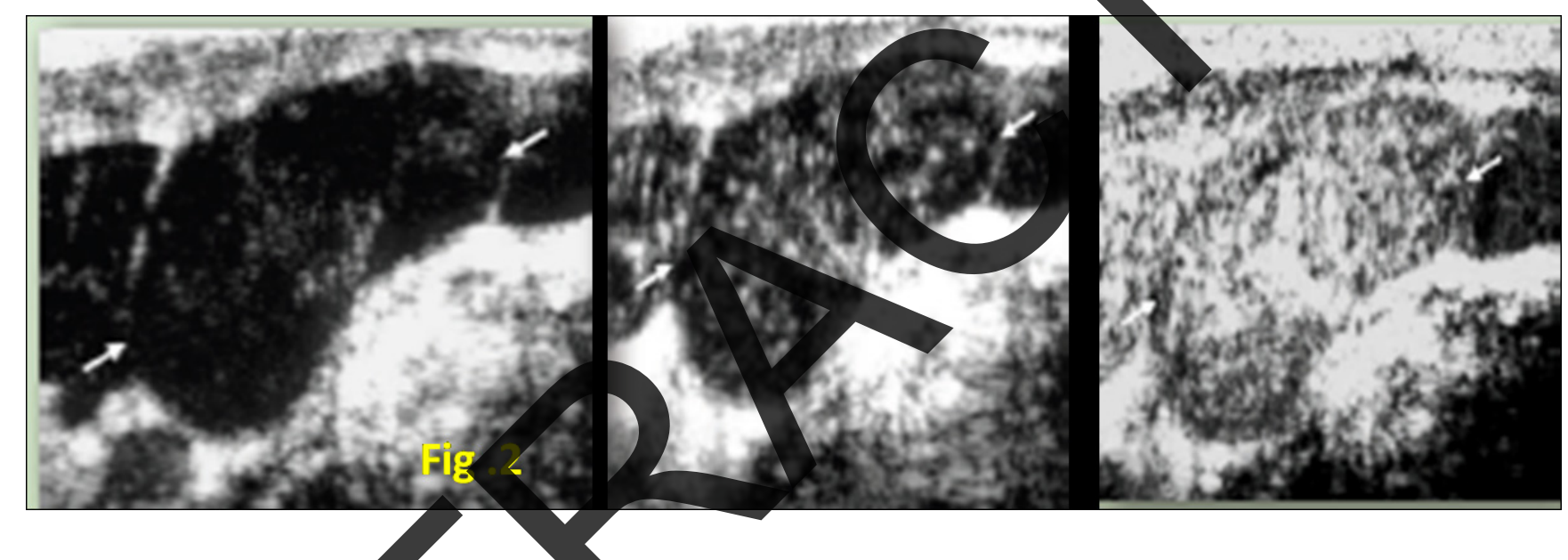

Figure 2: Lymphoma lymph node: CEUS shows inhomogeneous enhancement (snow storm) in the arterial phase and homogeneous in the parenchymal phase; In qut survey, only $15 \%$ showed similar behavior.

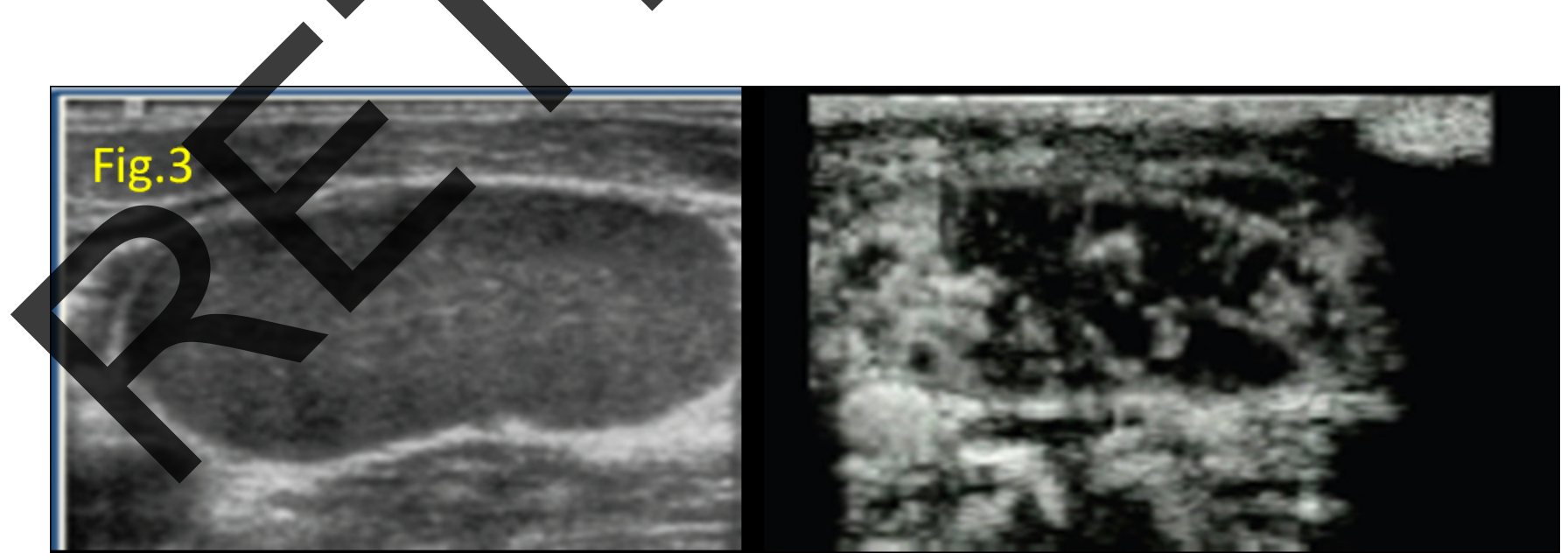

Figure 3: Tubercular lymph node CEUS shows a centripetal enhancement, intense but inhomogeneous enhancement in the arterial phase, due to the presence of colliquate areas. 
Table 3: Indicators of diagnostic accuracy using categories 1.2 and 3.4 with CEUS and Doppler

\begin{tabular}{|c|c|c|c|c|c|c|}
\hline & \multicolumn{2}{|c|}{ Histology } & & & & \\
\hline & & & sensitivity & specificity & $L R+$ & $V P P$ \\
\hline CEUS & \begin{tabular}{|l} 
Benign \\
$(61)$
\end{tabular} & $\begin{array}{l}\text { Malignant } \\
(50)\end{array}$ & & & & \\
\hline Positive & 9 & 36 & $72.0(58.3-82.5)$ & $85.2(74.2-92.0)$ & $4.8(2.6-9.1)$ & $80.0(65.4-90.4)$ \\
\hline Negative & 52 & 14 & & & & \\
\hline Doppler & $\begin{array}{l}\text { Benign } \\
\text { (61) }\end{array}$ & $\begin{array}{l}\text { Malignant } \\
(50)\end{array}$ & & & & \\
\hline Positive & 13 & 23 & $46.0(32.9-59,6)$ & $78.6(66.8-87.1)$ & $2.15(1.22$ & \\
\hline Negative & 48 & 27 & & & & \\
\hline
\end{tabular}

by a tumor, without alteration of the morphologic characteristics and with a low flow velocity of blood in the intranodal blood vessels. $[11,12]$

CEUS was used for the study of enlarged superficial lymph nodes because ultrasonographic contrast agents have a micro-bubble nature that is able to access all parts of the vascular system. Thus, CEUS is clinically valuable in microvascular applications focusing on the microcirculation patterns of the tissues.[12, 13]

Rubaltelli et al also investigated lymph nodes wi focal cortical thickening and documented a high specificity of CEUS, confirmed by histopathology.

In our study the sensitivity and lower than those reported by Rubaltelli but we hac results for enhancement patterns.

In 23 of 45 benign lymp 2 or less, and in 20 of 47 malignant lymph nodes the ratio was 2 or less. Yu et al showed that the L/T ratio is one of the less valuable parameters in the evaluation of lymph nodes and that a value of 2 or less has a low specificity and accuracy. We found that 45/ 6l benign lymph nodes showed a homogeneous enhancement at CEUS (7/61 benign nodes had a moderate enhancement), 8 benign ymph nodes showed inhomogeneous enhancement and had no enhancement. Thirty-two out of 50 malignant ymph nodes showed an inhomogeneous enhancement and 4 malignant nodes had no enhancement. Eight lymph nodes relating to lymphoma showed an intense homogeneous enhancement. Twelve out of 16 tubercular lymph nodes had an inhomogeneous pattern and 1 had no enhancement. The others appeared as reactive lymph nodes.

Benign enlarged lymph nodes have intense vascularization with a rich cortical capillary circulation, which would explain the homogeneous pattern.

Malignant lymph nodes are generally less vascularized and have perfusion defects or regions of necroses, which would explain the lack of blood flow and

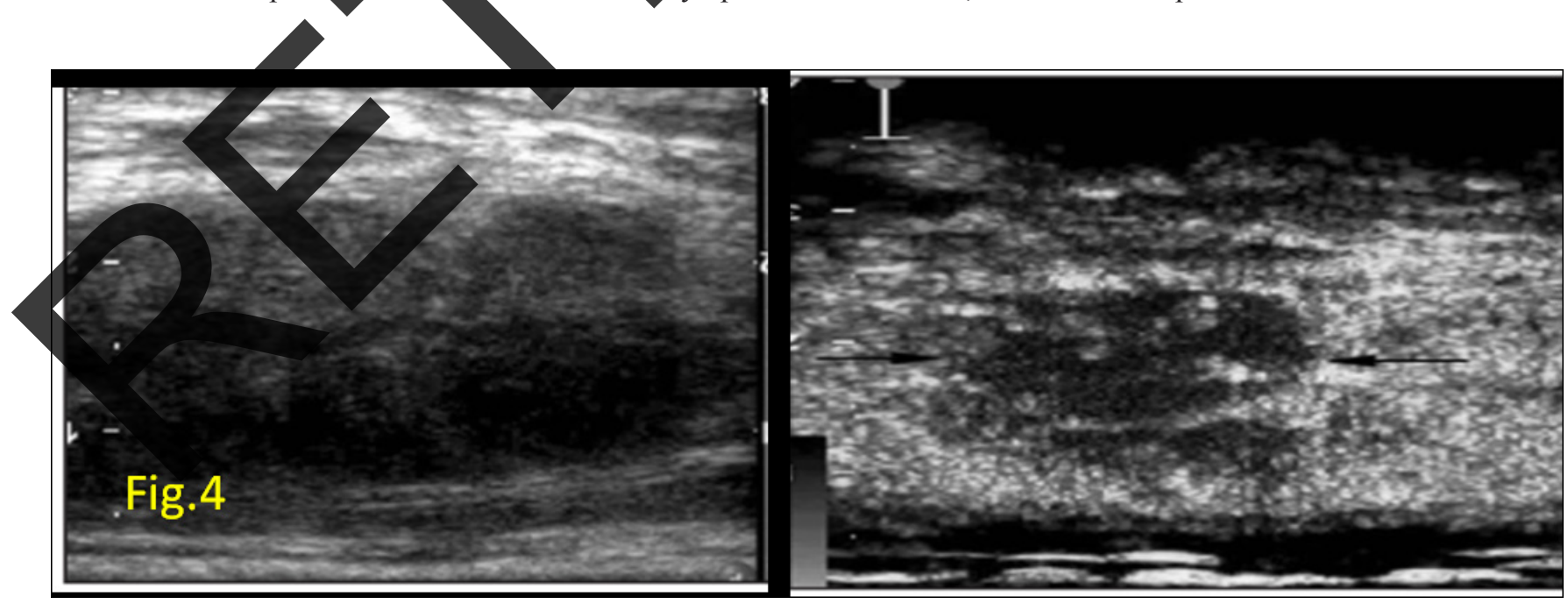

Figure 4: Metastatic lymph node CEUS shows an enhancement deficit due to reduced vascularity of the tumor area compared to the surrounding healthy tissue. 
inhomogeneous pattern.[14, 15]

Tubercular lymph nodes have a varied vascular pattern: displaced vascularity and apparent avascularity are common, relating to the high incidence of cystic necrosis.[15]

We found that many tubercular lymph nodes $(9 / 16)$ had a pattern typical of malignant nodes, due to areas of necrosis. These findings were observed in HIV-positive subjects.

Lymph nodes relating to lymphomas showed highly variable enhancement patterns.[16, 17] In our study, the most frequent pattern observed was homogeneous enhancement, 14/18 lymph nodes, and among these, 6 showed moderate homogeneous enhancement with a "snow storm" aspect, as described by Rubaltelli et al; only
3 lymph nodes had an inhomogeneous pattern and 2 nodes had no enhancement. In particular, an interesting finding was that the CEUS appearance of T-cell lymphoma fell into categories 1 and 2, whereas the CEUS appearance of B-cell lymphoma fell into categories 3 and 4 . The patients with B-cell lymphoma were HIV-positive.

The majority of lymphoma lymph nodes showed a preserved hilum vessel architecture resembling nonmalignant nodes.

\section{CONCLUSIONS}

In our study we identified four enhancement patterns in benign and malignant lymph nodes and lymphoma and tubercular nodes, with a high degree of diagnostic

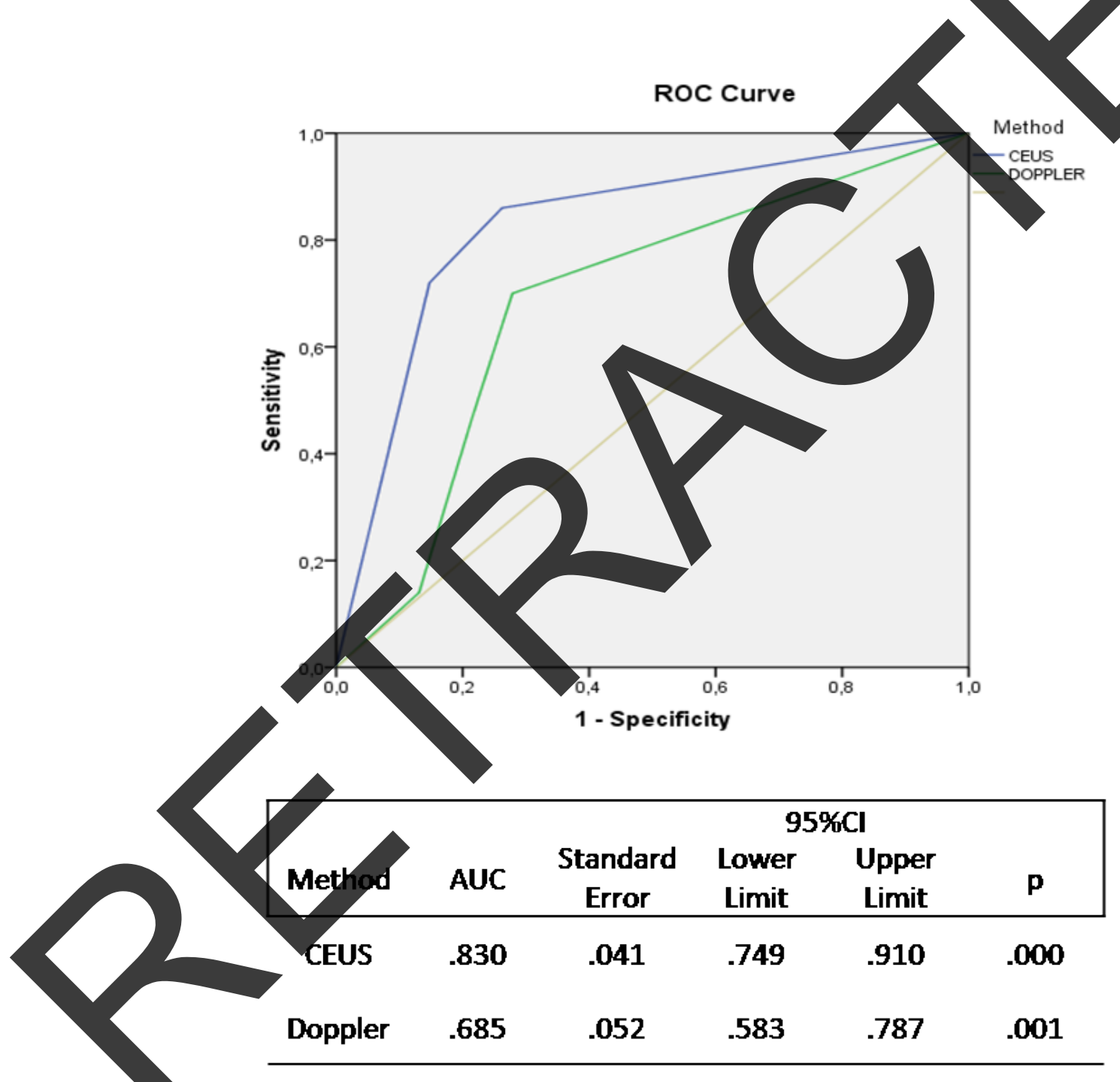

DeLong's test for two comelated ROC curves : $p<0.0001$

Figure 5 : ROC curve for the assessment of the diagnostic value of four patterns of lymph node perfusion for the CEUS and Doppler methods. 
accuracy for superficial lymphadenopathy in comparison with conventional US. CEUS demonstrated high spatial resolution, which is important for early detection of malignant lymph nodes, and improves the visualization of vessels, which is essential for the evaluation of vessel distribution. The visualization of avascular necrotic areas is helpful to distinguish benign from malignant lymphadenopathy and for the detection of lymph nodes of tubercular origin. Moreover, the injection of contrast agents allows the assessment of neoangiogenesis, which is of importance for treatment evaluation. CEUS cannot be recommended for the diagnosis of lymphoma, as yet, but it can be helpful to manage the response to therapy. Additional investigations are required to determine the ultimate benefit of CEUS in the assessment of patients with enlarged lymphadenopathy.

\section{CONFLICTS OF INTEREST}

None declared.

\section{REFERENCES}

1. Vassallo P, Wernecke K, Roos N, Peters PE.. Differentiation of benign and malignant superficial lymphadenopathy: the role of high-resolution US. Radiology 1992; 183: 215-220.

2. Dudea SM, Lenghel M, Botar-Jid C, Vasilescu D, D M. Ultrasonography of superficial lymph nodes: benign malignant. $2012 ; 14(4)$ :294-306.

3. Solbiati L1, Cioffi V, Ballarati E. Ultrasonography of the neck. Radial Clin North Am 1992; 30:

4. Cui XW1, Jenssen C, Saftoiu A, Ign ultrasound techniques for lymph Gastroenterol. $2013 ; 19(30)$ : i30.4850.

5. A.T. Ahuja, M. Ying King, and K.T. Wonga. Ultrasound of malignant cervical lymph nodes. Cancer Imaging 2008; 8. 48-56.

6. Slaisova R, Benda K, Jarkovsky J, Petrasova H, Szturz P, Valek Y. Contrast-enhanced ultrasonography compared to gray-scale and powerdoppler in the diagnosis of peripheral

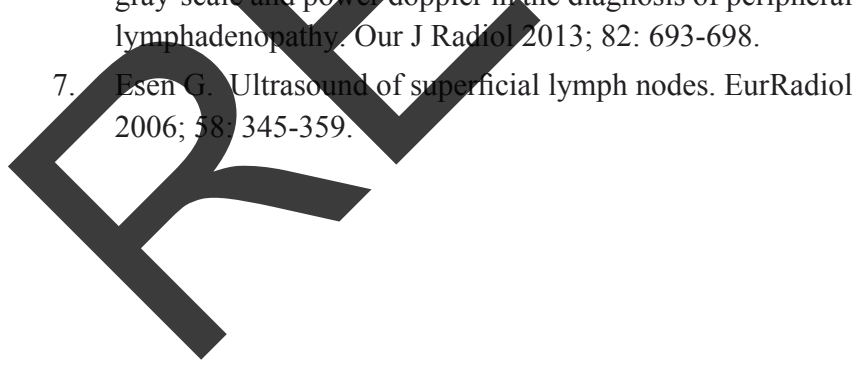

8. Poanta L, Serban O, Pascu I, Pop 2, Cosgarea M, Fodor D. The place of CEUS in distinguishing benign from malignant cervical lymph nodes: a prospective study. Med Ultrason 2014; 16(1): 7-14.

9. Liu GJ, Lu MD, Xie XY, Xu HX, Xu ZF, Zheng YL, Liang JY, Wang W. Real-time contrast-enhanced ultrasound imaging of infected focal liver lesions. J Ultrasound Med 2008; 27: 657-666.

10. Giovagnorio F, Galluzzo M, Andreoli C De CM, Devid V. Color Doppler sonography in the evaluation of superficial lymphomatous lymph nodes. J Ulrasound Med 2002; 21; 403-408.

11. Rubaltelli L, Khadivi Y

F, Borsato S, Fiocco of lymph node perfusion using continuous mode harmonic ultrasonography with a second generation contrast agent. J Ultrasound Med 2004; 23: 829-836.

12. Rubaltelli L, Beltrame V, Tregnaghi A, Scagliori E, Frigo AC, Stramare R. Contrast-enhanced ultrasound for characterizing lymph nodes with focal cortical thickening in patients with cutanequs melanoma. AJR Am J Roentgenol 2011;196: W8- W12.

Rubaltelli L,Proto E, Salmaso R, Bortoletto P, Candiani F, Cagol P. Sonography of abnormal lymph nodes in vitro: correlation of sonographic and histologic findings. AJR Am Roentgenol 1990; 155: 1241-1244.

14. Balázs-Đóme, Mary J.C. Hendrix, Sándor Paku, József Tóvári, and József Tímár. Alternative vascularization mechanism in cancer: pathology and therapeutic implications. Am J Pathol 2007; 170: 1-15.

15. Ouyang Q, Chen L, Zhao H, Xu R, Lin Q. Detecting metastasis of lymph nodes and predicting aggressiveness in patients with breast carcinomas. J Ultrasound Med 2010; 29: 343-352.

16. Castelijns JA, van den Brekel MW. Imaging of lymphadenopathy in the neck. Our Radial 2002; 12: 727738.

17. Stephanie R. Wilson1, Lennard D. Greenbaum2 and Barry B. Goldberg3 Contrast-enhanced Ultrasound: what is the evidence and what are the obstacles? AJR Aam J Roentgenol 2009; 193: 55-60. 